\title{
Shear-induced dynamics of polymeric globules at adsorbing homogeneous and inhomogeneous surfaces
}

\author{
Matthias Radtke ${ }^{\mathrm{a}}$ and Roland R. Netz \\ Fachbereich Physik, Freie Universität Berlin, Arnimallee 14, 14195 Berlin, Germany
}

Received 20 January 2014 and Received in final form 13 March 2014

Published online: 31 March 2014

(c) The Author(s) 2014. This article is published with open access at Springerlink.com

\begin{abstract}
The dynamics and adsorption behavior of a single collapsed homopolymer on a surface in shear flow is investigated by means of Brownian hydrodynamics simulations. We study different homogeneous and inhomogeneous surface models and determine dynamic state diagrams as a function of the cohesive strength, the adhesive strength, and the shear rate. We find distinct dynamical adsorbed states that are classified into rolling and slipping states, globular and coil-like states, as well as isotropic and prolate states. We identify two different cyclic processes based on trajectories of the polymer stretching and the polymer separation from the surface. For adsorption on an inhomogeneous surface consisting of discrete binding sites, we observe stick-roll motion for highly corrugated surface potentials. Although the resulting high surface friction leads to low drift velocities and reduced hydrodynamic lift forces on such inhomogeneous surfaces, a shear-induced adsorption is not found in the presence of full hydrodynamic interactions. A hydrodynamically stagnant surface model is introduced for which shear-induced adsorption is observed in the absence of hydrodynamic interactions.
\end{abstract}

\section{Introduction}

The behavior of polymers near surfaces is not only of fundamental and technological relevance, but also plays an important role in many biological processes [1]. As most technological and biological applications involve dissipation, there is general interest in understanding nonequilibrium aspects such as the adsorption of macromolecules in shear flow [2-4]. The present study is motivated by recent investigations [5] on the von Willebrand factor, a blood protein involved in hemostasis [6]. Experimental findings suggest the unfolding and activation of the von Willebrand factor at elevated shear rates as they are found in smaller blood vessels or at constriction sites [5] and subsequent binding to the vessel wall, which initiates the coagulation process $[6,7]$. This represents an example of a biological regulation mechanism that presumably involves protein unfolding by a purely physical mechanism and therefore is of particular interest for polymer theory.

Many theoretical studies focused on the equilibrium adsorption of stiff and flexible polymer chains $[1,8,9]$; also the scenario of surface-grafted chains was considered in detail $[10,11]$. When considering non-equilibrium effects, e.g. by inclusion of simple shear-flow [12-14], it has been shown that hydrodynamic interactions and the presence

\footnotetext{
${ }^{\mathrm{a}}$ e-mail: radtkem@physik.fu-berlin.de
}

of a no-slip boundary is crucial and therefore has to be taken into account [15-18].

In contrast to the adsorption of polymers in good solvent conditions, only very few works focused on the nonequilibrium adsorption of collapsed polymers. In a pioneering study, Sing and Alexander-Katz considered the interplay of the shear-induced globule stretching and the adsorption transition in detail [19]. They found that catchbonds are instrumental for observing shear-induced globule adsorption at the surface. This raises the interesting question what the minimal ingredients are in order to observe the counter-intuitive phenomenon of shear-induced adhesion of a polymeric globule on a surface.

In the present paper, we approach this problem by studying the dynamical states of a single globular polymer in shear flow at an adsorbing surface that is modeled on different levels of resolution. Using Brownian dynamics simulations including hydrodynamic interactions, we map out detailed dynamic adsorption state diagrams for three different kinds of surface models: i) a homogeneous adsorbing surface, ii) a homogeneous surface with a stagnant boundary layer within which the monomer mobility is reduced, and iii) a laterally inhomogeneous surface consisting of discrete binding sites with varying potential range. In particular, we consider the dynamics of the adsorbed globule and distinguish rolling states from slipping deformed states. Our study can thus be viewed as an extension of previous works on the rolling characteristics of a 
non-deformable sphere on a surface with discrete binding sites [2]. We obtain new dynamic states where the shear forces deform the adsorbed polymer into a prolate shape; this occurs both for coil-like conformations at low cohesion as well as for globular conformation at high cohesion. As a result, we find rich dynamic state diagrams as a function of shear rate and varying adhesive and cohesive strength, featuring various rolling and slipping states of globular and coil-like configurations with either isotropic or prolate shape. With the advent of advanced microscopy techniques such shape changes can be conveniently observed experimentally $[5,20]$. In addition, we find different cyclic transformations depending on whether the globule is adsorbed or desorbed, indicative of fundamentally different surface-induced dissipative processes.

In the presence of hydrodynamic interactions we only observe shear-induced desorption of the polymeric globule from the surface; however, in the absence of hydrodynamic interactions, in which case shear-induced lift forces are absent, and for a hydrodynamically stagnant boundary layer, shear-induced adsorption is obtained. All our simulations are based on a potential-based Hamiltonian, meaning that the surface-polymer bonds correspond to slip-bonds. One conclusion from our work is that a necessary condition for shear-induced adsorption are hydrodynamic screening effects as well as catch-bond behavior between polymeric units and the surface, as was convincingly demonstrated by Sing and Alexander-Katz [19].

\section{Simulation details}

We perform Brownian dynamics simulations and incorporate hydrodynamic interactions via the Rotne-PragerBlake mobility tensor, which accounts for the no-slip surface boundary condition and the finite particle size via a multipole expansion to second order in terms of the particle radius [21,22]. This method is practical for small particle numbers and large system volumes, as we have in our study, and thus preferred over alternative LatticeBoltzmann methods [23] or multi-particle collision dynamics [24]. The discretized Langevin equation for particle $i$ and time step $\Delta t=\Delta \tilde{t} / \tau$ is given by

$$
\begin{aligned}
\mathbf{r}_{i}(t+\Delta t)= & \mathbf{r}_{i}(t)+\left(\boldsymbol{\mu}_{i i} \dot{\gamma} z_{i} \hat{\mathbf{x}}-\sum_{j=1}^{N} \boldsymbol{\mu}_{i j} \cdot \nabla_{\mathbf{r}_{j}} U(t)\right. \\
& \left.+\sum_{j} \nabla_{\mathbf{r}_{j}} \boldsymbol{\mu}_{i j}\right) \Delta t+\boldsymbol{\xi}_{i}(t) .
\end{aligned}
$$

Note that all quantities used are made dimensionless by rescaling lengths $r=\tilde{r} / a$ by the monomer radius $a$, energies $U=\tilde{U} / k_{B} T$ by thermal energy and times $t=\tilde{t} / \tau$ by the characteristic monomer diffusion time $\tau=a^{2} /\left(\mu_{0} k_{B} T\right)$, where $\mu_{0}$ is the Stokes mobility. The first term in parentheses in eq. (1) represents a linear shear flow with rate $\dot{\gamma}=\tilde{\dot{\gamma}} \tau$, where $\hat{\mathbf{x}}$ is the unit vector in the $x$ direction. Note that we do not consider elongational flow effects, which were shown to very efficiently promote chain unfolding $[25,26]$. The second term accounts for the direct force acting on particle $i$ itself as well as the hydrodynamic flow field created by forces acting on all other particles $j \neq i$. Hydrodynamic interactions are taken into account via the mobility matrix

$$
\boldsymbol{\mu}_{i j}=\tilde{\boldsymbol{\mu}}_{i j} / \mu_{0}=\boldsymbol{\mu}_{\mathrm{self}}^{\mathrm{RPB}}\left(z_{i}\right) \delta_{i j}+\left(1-\delta_{i j}\right) \boldsymbol{\mu}^{\mathrm{RPB}}\left(\mathbf{r}_{i}, \mathbf{r}_{j}\right)
$$

Explicit expressions for the self-mobilities $\boldsymbol{\mu}_{\text {self }}^{\mathrm{RPB}}\left(z_{i}\right)$ and the off-diagonal elements $\boldsymbol{\mu}^{\mathrm{RPB}}\left(\mathbf{r}_{i}, \mathbf{r}_{j}\right)$ have been given previously on the Rotne-Prager-Blake level [22]. The third term in eq. (1) compensates for the spurious flux due to inhomogeneities in the self-mobilities [27]. The stochastic contribution $\boldsymbol{\xi}_{i}$ is given by Gaussian random vectors with correlations according to the fluctuation-dissipation theo$\operatorname{rem}\left\langle\boldsymbol{\xi}_{i}(t) \boldsymbol{\xi}_{j}\left(t^{\prime}\right)\right\rangle=6 \boldsymbol{\mu}_{i j} \Delta t \delta\left(t-t^{\prime}\right)$ and vanishing mean. The simulation time step is typically chosen as $\Delta t=10^{-4}$ and for computational speed we update the mobility matrix $\boldsymbol{\mu}_{i j}$ only every tenth step, which does not alter the results.

The polymer consists of $N=50$ beads, which interact via Lennard-Jones potentials of depth $\varepsilon=\tilde{\varepsilon} / k_{B} T$ and are connected in a linear chain by stiff harmonic bonds with a rescaled spring constant $k=\tilde{k} /\left(k_{B} T a^{2}\right)=200$,

$U_{\text {pol }}=\varepsilon \sum_{i<j}\left(\left(2 / r_{i j}\right)^{12}-2\left(2 / r_{i j}\right)^{6}\right)+\frac{k}{2} \sum_{i}\left(r_{i, i+1}-2\right)^{2}$.

The total potential energy $U=U_{\text {pol }}+U_{\text {hom } / \text { inh }}+U_{\text {rep }}$ includes in addition a surface term that accounts for adhesion, $U_{\text {hom/inh }}$, and a term $U_{\text {rep }}$ that represents a purely repulsive wall.

The inhomogeneous surface iii) is modeled by a square lattice of discrete binding sites with distance $b$, located at a height $z_{W}=2$ above the no-slip boundary located at $z=0$. The rescaled surface density of binding sites is thus $\rho_{W}=\tilde{\rho}_{W} a^{2}=1 / b^{2}$. Summation over all pair interactions gives the surface potential as

$$
U_{\mathrm{inh}}=\sum_{i} \sum_{k} \varepsilon_{W}\left(\frac{\sigma_{W}^{12}}{\left(\sigma_{W}+r_{i k}\right)^{12}}-\frac{2 \sigma_{W}^{6}}{\left(\sigma_{W}+r_{i k}\right)^{6}}\right),
$$

where $r_{i k}$ is the distance between monomer $i$ and binding site $k$. We have shifted the potential by the range $\sigma_{W}=\tilde{\sigma}_{W} / a$ and therefore have a purely attractive potential with a depth of $\varepsilon_{W}=\tilde{\varepsilon}_{W} / k_{B} T$.

The homogeneous surface model i) follows from eq. (4) in the limit of high binding site density $\rho_{W}>\sigma_{W}^{-2}$ by replacing the sum by an integral, the resulting homogeneous surface potential reads

$$
\begin{aligned}
U_{\mathrm{hom}}= & \sum_{i} \frac{\pi}{5} \varepsilon_{W} \rho_{W} \sigma_{W}^{2}\left(\frac{\left(11\left|z_{i}-2\right|+\sigma_{W}\right) \sigma_{W}^{10}}{11\left(\sigma_{W}+\left|z_{i}-2\right|\right)^{11}}\right. \\
& \left.-\frac{\left(5\left|z_{i}-2\right|+\sigma_{W}\right) \sigma_{W}^{4}}{\left(\sigma_{W}+\left|z_{i}-2\right|\right)^{5}}\right) .
\end{aligned}
$$

To account for steric exclusion of the polymer from the surface and in particular to prevent crossing of the no-slip 


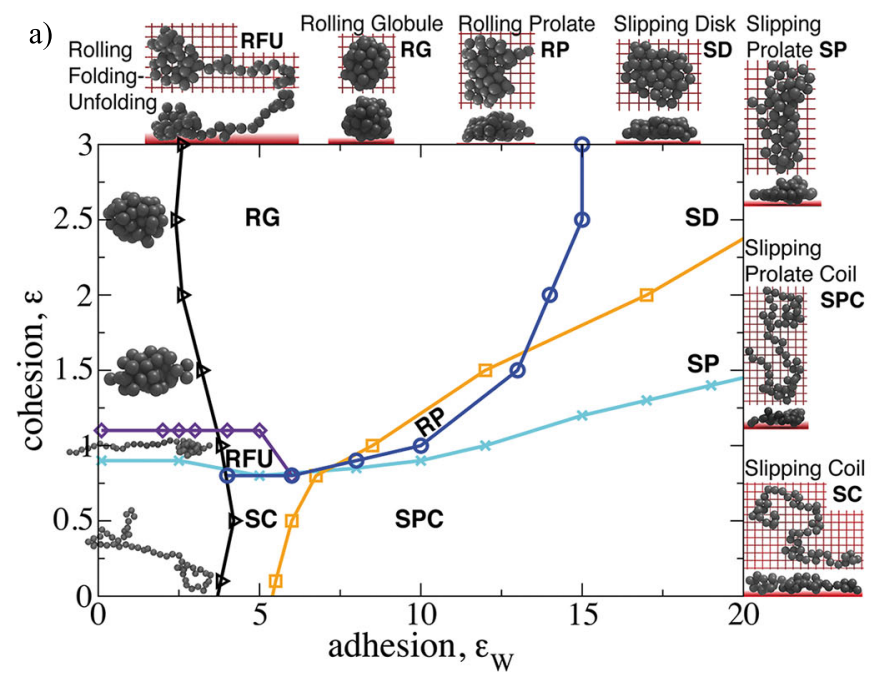

b)

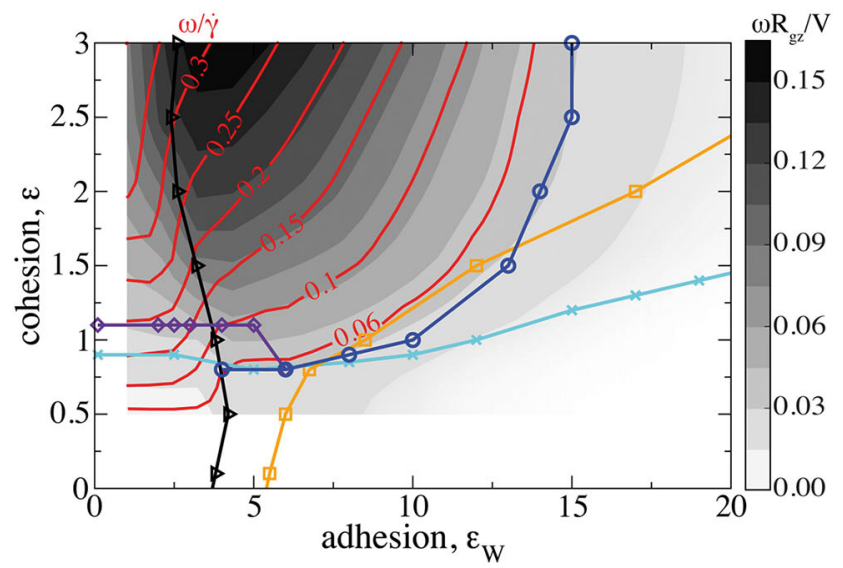

Fig. 1. Dynamical state diagram for a polymer with varying cohesive strength $\varepsilon$ and adhesive strength $\varepsilon_{W}$ at fixed shear rate $\dot{\gamma}=1$. The black line denotes the adsorption transition as determined by a distance criterion. The dark blue line marks the transition from rolling to slipping motion, determined by a value of the rescaled angular velocity of $\omega / \dot{\gamma}=0.05$ and for high $\varepsilon$ separates the rolling globule state (RG) from the slipping disk state (SD). In (b) the distribution of $\omega / \dot{\gamma}$ is illustrated by red contour lines, while the rolling parameter $\omega R_{g z} / V$ is given in shades of gray. The orange line denotes the transition into a prolate arrangement perpendicular to the flow direction and separates the slipping disk (SD) from the slipping prolate state (SP), the rolling globule (RG) from the rolling prolate (RP), and the slipping coil (SC) from the slipping prolate coil state (SPC). The collapse transition between the coil state at low $\varepsilon$ and the globular state at high $\varepsilon$ is denoted by the cyan line. The purple line is defined by a maximum in the chain extension fluctuations and separates the rolling globule (RG) from the rolling folding-unfolding state (RFU). Snapshots in (a) show representative conformations for each state.

boundary located at $z=0$, we use an additional repulsive potential

$U_{\text {rep }}=\sum_{i} \begin{cases}2 \pi \sigma_{R}^{2}\left(\frac{2}{5}\left(\frac{\sigma_{R}}{z_{i}}\right)^{10}-\left(\frac{\sigma_{R}}{z_{i}}\right)^{4}+\frac{3}{5}\right), & z_{i} \leq \sigma_{R}, \\ 0, & z_{i}>\sigma_{R},\end{cases}$

with a short range of $\sigma_{R}=1.2$. Furthermore, we prevent escape of the polymer to infinity by a soft wall acting on the polymer center of mass located at a height $z=15$.

Surface model ii) employs a homogeneous surface potential and is characterized by a hydrodynamically stagnant boundary layer within which the monomer self-mobility is reduced. We thus replace the Rotne-Prager-Blake self-mobility profile $\boldsymbol{\mu}_{\mathrm{self}}^{\mathrm{RP}}\left(z_{i}\right)$ by the heuristic expression

$$
\mu_{\text {self }}\left(z_{i}\right)=\left(1-\mu_{s}\right)\left[\tanh \left(10\left(z_{i}-2.5\right)\right)+1\right] / 2+\mu_{s},
$$

which yields a rapid yet smooth transition from the bulk mobility $\mu_{\text {self }}\left(z_{i} \rightarrow \infty\right)=1$ to a reduced surface mobility $\mu_{s}=\tilde{\mu}_{s} / \mu_{0}$ at around a distance $z=2.5$. Equation (7) constitutes a simple heuristic model to investigate surface friction effects that might for example be due to discrete surface binding sites or hydrodynamic screening caused by surface corrugations or grafted polymers.

\section{Results for homogeneous surface}

\subsection{Fixed shear rate, variable cohesive strength}

The range of the homogeneous surface potential eq. (5) is set to $\sigma_{W}=1.5$ and the density is fixed at $\rho_{W}=0.25$. We first present the dynamic state diagram for varying cohesive strength $\varepsilon$ and adhesive strength $\varepsilon_{W}$ at a fixed shear rate $\dot{\gamma}=1$ in fig. 1 . All different dynamic states are illustrated by representative snapshots in fig. 1a. The black almost vertical line denotes the transition from the desorbed state for small adhesion $\varepsilon_{W}$ (to the left) to different adsorbed states for large adhesion $\varepsilon_{W}$ (to the right). Various heuristic definitions have been proposed for this transition, which for a finite-length polymer does not correspond to a phase transition in the strict thermodynamic sense. We here use for the adsorbed state the simple distance criterion $\left(z_{\mathrm{COM}}-R_{g z}\right)<\left(z_{W}+\sigma_{W}\right)$, where $z_{\mathrm{COM}}=N^{-1} \sum_{i}^{N} z_{i}$ is the polymer center-of-mass height, $R_{g z}^{2}=N^{-1} \sum_{i}^{N}\left(z_{i}-z_{\mathrm{COM}}\right)^{2}$ is the $z$-component of the radius of gyration. Our criterion thus defines the polymer to be adsorbed when the polymer height $z_{\mathrm{COM}}$ corrected by the radius $R_{g z}$ is smaller than the sum of the potential range $\sigma_{W}$ and the location of the potential origin $z_{W}$. In fig. $2 \mathrm{a}$ we plot the average $z_{\mathrm{COM}}-z_{W}$ for different values of $\varepsilon$ as a function of the adhesion $\varepsilon_{W}$, the transition according to our criterion is denoted by broken vertical lines. The time series of $z_{\mathrm{COM}}$ for $\varepsilon=1$ and $\varepsilon_{W}=3.2 \mathrm{in}$ fig. 3a demonstrate that the polymer alternates between 

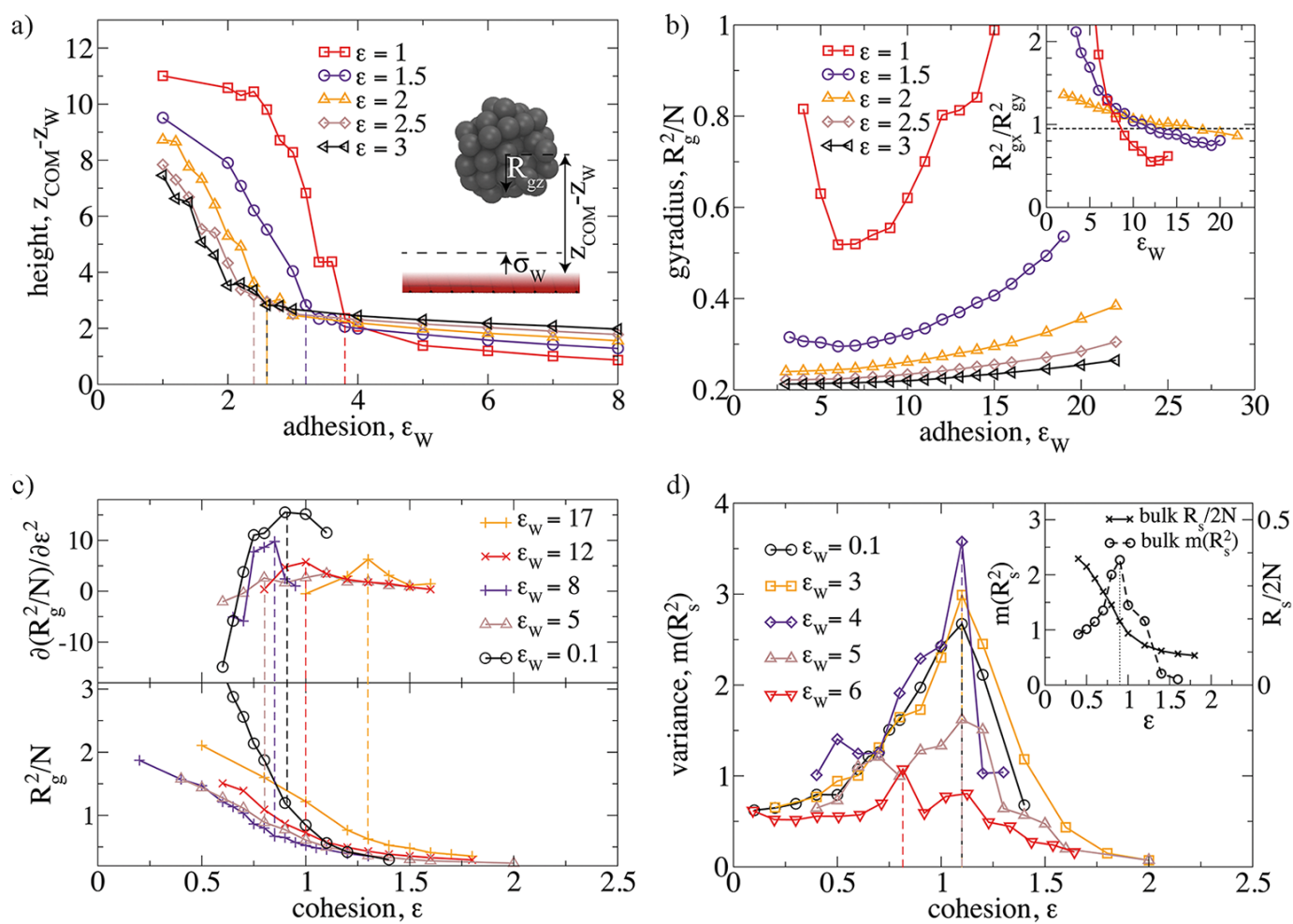

Fig. 2. a) Average height of the polymer center of mass $z_{\mathrm{COM}}$ relative to the surface location $z_{W}$ for varying adhesive strength $\varepsilon_{W}$ and different fixed values of the cohesive strength $\varepsilon$. The adsorption transition defined by $\left(z_{\mathrm{COM}}-z_{W}\right)=\left(R_{g z}+\sigma_{W}\right)$ is indicated by vertical dashed lines, where $R_{g z}$ is the $z$-component of the radius of gyration and $\sigma_{W}$ is the surface interaction range, see snapshot. b) Squared radius of gyration $R_{g}^{2} / N$ as a function of $\varepsilon_{W}$. The inset shows the ratio of the squared components $R_{g x}^{2}$ and $R_{g y}^{2}$ parallel and perpendicular to the flow direction. The horizontal dashed line indicates the prolate-isotropic transition defined by $R_{g x}^{2} / R_{g y}^{2}=0.95$. c) Squared radius of gyration $R_{g}^{2} / N$ and its curvature $\partial^{2}\left(R_{g}^{2} / N\right) / \partial^{2} \varepsilon$ as a function of $\varepsilon$. The maxima of the curvature define the collapse transition and are denoted by vertical dashed lines. d) Variance of the squared extension in flow direction $m\left(R_{S}^{2}\right)$ for varying $\varepsilon$. The peaks define the stretch transition (dashed vertical lines). The same quantity for a polymer in bulk is shown in the inset.

desorbed and adsorbed states, according to our distance criterion the adsorption occurs for $\varepsilon=1$ around $\varepsilon_{W}=3.8$.

In the absence of hydrodynamic boundaries, an isolated hard sphere in simple shear rotates with a frequency of $\omega=\dot{\gamma} / 2$; we in fact verified that our hydrodynamic simulation code reproduces this limiting result for large values of the cohesion $\varepsilon$ (data not shown). When a hard sphere approaches a surface with a hydrodynamic no-slip boundary condition, the rotational frequency is reduced due to lubrication effects but reaches a finite value upon contact [28]. For deformable objects such as polymers or vesicles, the rotation frequency is reduced additionally due to dissipative effects associated with periodic stretching and relaxation cycles $[20,29]$. Note that on a homogeneous surface, we always find substantial slip of the globule on the surface since the monomer self-mobility decreases rather smoothly as the surface is approached (this is different on an inhomogeneous surface where additional friction effects are produced by lateral surface-monomer interactions, as we will discuss in sect. 5). The rotation in the rolling globule state $(\mathrm{RG})$ is illustrated in fig. $1 \mathrm{~b}$ by contour lines of the rescaled angular velocity $\omega / \dot{\gamma}=L_{y} /\left(J_{y} \dot{\gamma}\right)$, where $L_{y}$ and $J_{y}$ are the polymer angular momentum and moment of inertia with respect to the $y$-axis, respectively. We see that for large cohesion $\varepsilon$ and small adhesion $\varepsilon_{W}$ rotation is pronounced. In fig. $1 \mathrm{~b}$ we also show a contour plot of the rolling parameter defined by the ratio $\omega R_{g z} / V$, where $V$ denotes the center-of-mass velocity. For a compact object that rolls without slip over a surface the rolling parameter is of order unity. One sees that as $\varepsilon_{W}$ increases both $\omega / \dot{\gamma}$ and $\omega R_{g z} / V$ decrease in a very similar way, leading to the conclusion that rolling and rotation in the adsorbed globular state is strictly coupled. We define the transition from rolling to slipping for $\omega / \dot{\gamma}=0.05$, which corresponds to ten percent of the bulk value and is denoted by a dark blue line.

At high cohesion $\varepsilon$ the rolling globule state $(\mathrm{RG})$ yields to the slipping disk state (SD) as the adhesion $\varepsilon_{W}$ increases, where the polymer is still very compact but flattened out on the surface (see snapshot). The discoid shape in the SD state prevents rolling and rotation. As the cohesion $\varepsilon$ is reduced at relatively high adhesion $\varepsilon_{W}$, the shear forces deform the polymer and give rise to a slipping prolate state (SP) at high $\varepsilon_{W}$ and, in a very small window for intermediate values of $\varepsilon_{W}$, to a rolling prolate state $(\mathrm{RP})$. We define the transition from the symmetric 


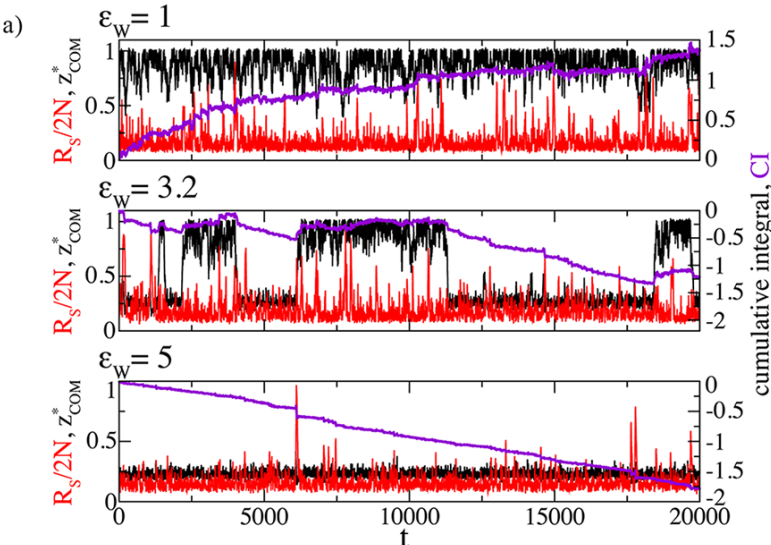

b)

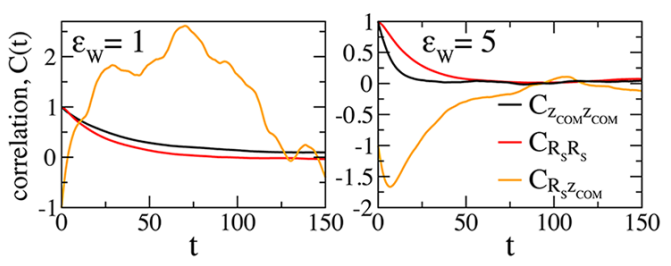

c)
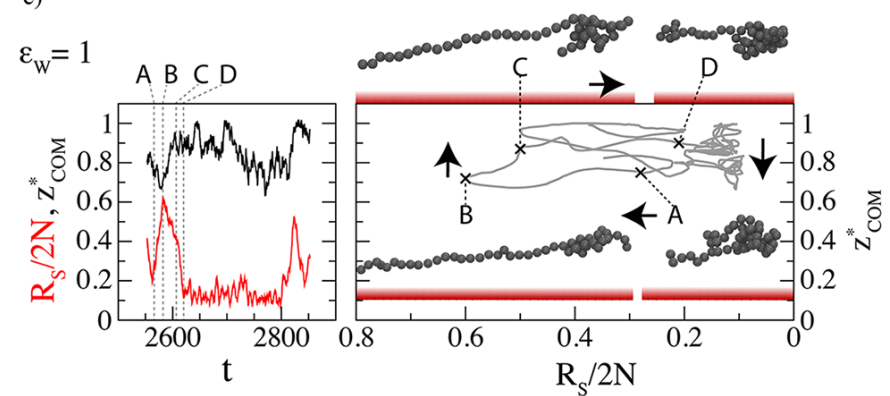

d)

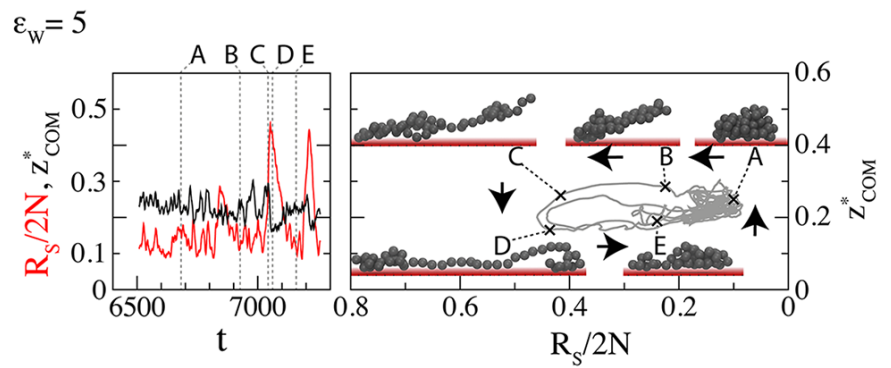

Fig. 3. a) Time series of the rescaled extension $R_{S} / 2 N$ (red) and of the center-of-mass separation from the surface rescaled by the simulation box height $z_{\mathrm{COM}}^{*}=z_{\mathrm{COM}} / 15$ (black) are compared for fixed cohesion $\varepsilon=1$ and fixed shear rate $\dot{\gamma}=1$ for different adhesive strength values. In the desorbed state for $\varepsilon_{W}=1$ polymer stretching occurs, indicated by peaks in $R_{S}$, when the polymer is located close to the surface. The cumulative integral CI (purple) of $R_{S}$ versus $z_{\mathrm{COM}}^{*}$ increases over time. Close to the adsorption transition at $\varepsilon_{W}=3.2$, the polymer is only transiently adsorbed on the surface and CI shows alternating behavior. For $\varepsilon_{W}=5$ the polymer is firmly adsorbed, stretching events are rare and CI monotonically decreases over time. b) Auto-correlation and cross-correlation functions of extension and height are shown for $\varepsilon_{W}=1$ and $\varepsilon_{W}=5$. c) One typical cycle (gray line) of a desorbed polymer for $\varepsilon_{W}=1$ is shown and illustrated with snapshots, corresponding to adjacent labels. The cycle is traversed clockwise, leading to a positive increase of the cumulative integral CI. d) When the polymer is adsorbed for $\varepsilon_{W}=5$, the cycle is traversed counter-clockwise, hence CI decreases in time.

to prolate shapes using the radius of gyration components parallel and perpendicular to the shear flow. If the ratio $R_{g x}^{2} / R_{g y}^{2}$ is smaller than 0.95 we denote the structure as prolate. In fig. 1 this structural transition is denoted by an orange line and illustrated in the inset of fig. $2 \mathrm{~b}$, where we plot $R_{g x}^{2} / R_{g y}^{2}$ as a function of $\varepsilon_{W}$ for a few different values of $\varepsilon$.

In the lower left corner of the rolling region in fig. 1 we find a rolling folding-unfolding (RFU) state, which is characterized by cyclic stretching-refolding events combined with a rolling motion. The purple line is defined by the maximum of the variance of the squared extension $R_{S}^{2}$ in flow direction, as illustrated in fig. $2 \mathrm{~d}$, and is generally referred to as a shear-induced unfolding transition [30,31]. The extension $R_{S}$ is defined as the maximal distance in flow direction between any two beads from which the normalized variance follows as

$$
m\left(R_{S}^{2}\right)=\left(\left\langle R_{S}^{4}\right\rangle-\left\langle R_{S}^{2}\right\rangle^{2}\right) /\left\langle R_{S}^{2}\right\rangle^{2} .
$$

The unfolding transition between the RG and RFU states continuously extends into the desorbed region, which is related to the fact that we limit the polymer separation from the surface by the finite height of the simulation box. Note that in the absence of a bounding wall, i.e. in bulk, this transition occurs at a somewhat lower cohesion of $\varepsilon=0.9$ as illustrated in the inset of fig. $2 \mathrm{~d}$. This surfaceinduced unfolding enhancement has been demonstrated previously [32].

In general, we discriminate coil from globule conformations by the magnitude of the squared radius of gyration $R_{g}^{2}$, the collapse transition is denoted by the cyan line in fig. 1 and defined by the maximum curvature of $R_{g}^{2}$, as illustrated in fig. 2c. Note that we make a fine distinction between the collapse transition, associated with the radius of gyration, and the shear-induced unfolding transition, linked to the chain extension in flow direction. It turns out that an adsorbed coil is always slipping, meaning that no or only very little solid-body rotation occurs. This becomes most transparent from the red iso-rotationalfrequency contour lines in fig. 1b. The transition between isotropic and prolate conformations (orange line) extends into the coil state and separates the slipping isotropic coil state SC from the slipping prolate coil state SPC, in agreement with previous observations $[13,33]$. In fig. $2 \mathrm{~b}$ we plot $R_{g}^{2}$ for different values of $\varepsilon$ as a function of $\varepsilon_{W}$. In the rolling globular (RG) state, i.e. for high values of $\varepsilon, R_{g}^{2}$ increases monotonically due to the globule deformation caused by the strongly attractive surface. The case $\varepsilon=1$ is different since we cross the rolling folding-unfolding (RFU) 
region, see the state diagram fig. 1 . Here the radius of gyration first decreases with growing adhesion $\varepsilon_{W}$ since the attractive wall suppresses the periodic folding-unfolding cycles, for larger $\varepsilon_{W}$ we see that $R_{g}^{2}$ increases again because the globule flattens out on the surface.

\subsection{Cyclic transformations}

We now analyze the non-equilibrium polymer dynamics in shear flow in more detail and in particular discuss the occurrence of cyclic processes. In fig. 3a we plot time series of the rescaled extension $R_{S} / 2 N$ (red) and the centerof-mass position $z_{\mathrm{COM}}^{*}=z_{\mathrm{COM}} / 15$ (black) rescaled by the height of the simulation box; note that these rescaled variables are strictly bound by unity. In the same graph we also display the cumulative integral

$$
\mathrm{CI}(t)=\int_{0}^{t} \frac{R_{S}\left(t^{\prime}\right)}{2 N} \mathrm{~d} z_{\mathrm{COM}}^{*}\left(t^{\prime}\right),
$$

as a purple line. For the desorbed polymer with $\varepsilon_{W}=1, \mathrm{CI}$ increases in time, for the adsorbed polymer with $\varepsilon_{W}=5$, CI decreases, and for the intermediate situation $\varepsilon_{W}=$ 3.2 we see an intermediate behavior where CI decreases over periods where the polymer is adsorbed (and $z_{\mathrm{COM}}^{*}$ is small) and otherwise stays constant or slightly increases.

Intuitively speaking, CI measures the enclosed area of the trajectory of $R_{S}(t) / 2 N$ versus $z_{\mathrm{COM}}^{*}(t)$ which is shown in fig. $3 \mathrm{c}$,d for two different values of the adhesive strength $\varepsilon_{W}$. For the desorbed state with $\varepsilon_{W}=1$ in fig. 3c, we see that an increasing CI means that the trajectory corresponds to predominantly clockwise cyclic transformations. The mechanism for this can be visualized by typical chain snapshots: When the polymer diffuses towards the surface in a rather compact state, i.e. small $R_{S}$, see snapshot (A), surface-induced stretching occurs because of the decreased rotational and translational polymer mobility at the surface, leading to state (B), as has been discussed before [32]. The stretched form gives rise to an increased hydrodynamic lift force away from the surface, and thus induces the transition to state $(\mathrm{C})$. Away from the surface, the unfolding tendency is smaller, which leads to a refolding and thus to a transition from (C) to (D). In the adsorbed state for $\varepsilon_{W}=5$ the cycle is traversed predominantly counter-clockwise, in fig. $3 \mathrm{~d}$ we show a typical cycle. In (A) the adsorbed globule has a rather compact state. A partial unfolding is initiated (B-C) by a protrusion [30], leading to a polymer strand that is highly stretched by hydrodynamic drag forces and oriented parallel to the surface. Since the stretched chain shows reduced fluctuations in the perpendicular direction to the surface, it is more easily adsorbed leading to state (D) where the center-ofmass height is reduced compared to the globular state. This stretching-induced adsorption mechanism has been previously discussed in the absence of hydrodynamic interactions and is typically overwhelmed by hydrodynamic drag effects $[34,17]$, in the present situation it seems to be operative since the chain is inhomogeneous and consists of a stretched protrusion and a globular section that acts as an anchor. Once adsorbed, the shear forces are reduced and the polymer can refold into a globular state (E), this transformation is assisted by the rolling motion of the chain, and leads back to the starting configuration of a globule adsorbed at the surface, state (A). Close to the adsorption transition $\varepsilon_{W}=3.2$ in fig. $3 \mathrm{a}$, the polymer alternates between clockwise and counter-clockwise trajectories for the desorbed and the adsorbed state and thus the cumulative integral is closer to zero.

This picture can be substantiated by the correlation function of two variables $A(t)$ and $B(t)$ defined as

$$
C_{A B}(\tau)=(\langle A(t) B(t+\tau)\rangle-\langle A\rangle\langle B\rangle) /\left(\sigma_{A} \sigma_{B}\right),
$$

where $\sigma_{A, B}$ are the respective standard deviations. We show in fig. $3 \mathrm{~b}$ auto- and cross-correlations for $R_{S}$ and $z_{\mathrm{COM}}$ for the desorbed $\varepsilon_{W}=1$ and the adsorbed $\varepsilon_{W}=5$ states. While the autocorrelation functions $C_{R_{S} R_{S}}(\tau)$ and $C_{z_{\mathrm{COM}} z_{\mathrm{COM}}}(\tau)$ are positive and decay in a roughly exponential manner both in the adsorbed and desorbed states, the cross-correlation function $C_{R_{S} z_{\mathrm{COM}}}(\tau)$ has a different sign in the two states and decays over a rescaled time of about 100 , which is roughly the time span of the cycles shown in fig. $3 \mathrm{c}$ and $\mathrm{d}$.

\subsection{Fixed cohesive strength, variable shear rate}

In fig. 4a we plot the dynamic state diagram on a homogeneous surface as a function of the adhesive strength $\varepsilon_{W}$ and the shear rate $\dot{\gamma}$ for fixed cohesive strength $\varepsilon=2$, in which case the polymer forms a well-defined globule for low shear flow. The desorption-adsorption transition, obtained from the mean separation of the polymer from the adsorbing surface as explained before, is marked by a black line. As a main observation we see that the higher the shear rate, the more adhesive strength is needed to adsorb the globule. A marked change in the slope of this transition is seen around $\varepsilon_{W}=2.5$, i.e., where the adhesion is of comparable strength as the cohesion. For large adhesion, the critical shear rate scales roughly linear with the adhesive strength $\varepsilon_{W}$. This suggests that the hydrodynamic lift force that is responsible for the desorption at high shear scales linear in the shear rate $\dot{\gamma}$ and is caused by a stationary disk-like deformation of the globule shape. For small adhesion the critical shear rate rises much more steeply with adhesive strength $\dot{\gamma} \sim \varepsilon_{W}^{6}$; this regime is more intricate as the shear-induced lift force presumably depends via the adhesion-induced globule deformation also on the adhesive strength $\varepsilon_{W}$. As before, we define the transition between rolling globule (RG) and slipping disk (SD) states by a critical value of the angular velocity of $\omega / \dot{\gamma}=0.05$ denoted by the blue line. Increasing the adhesive strength $\varepsilon_{W}$ flattens the globule and leads to the SD state. In the adhesion range $9<\varepsilon_{W}<16$ we see slip reentrance as the shear rate $\dot{\gamma}$ is increased: Only for intermediate values of $\dot{\gamma}$ does the shear induce rolling, for higher shear rates and close to the desorption transition the increased stretching in the flow direction inhibits rolling of the chain.

The squared average extension $R_{s}^{2}$ in fig. $4 \mathrm{~b}$ monotonically increases with shear rate, the stretch-transition 

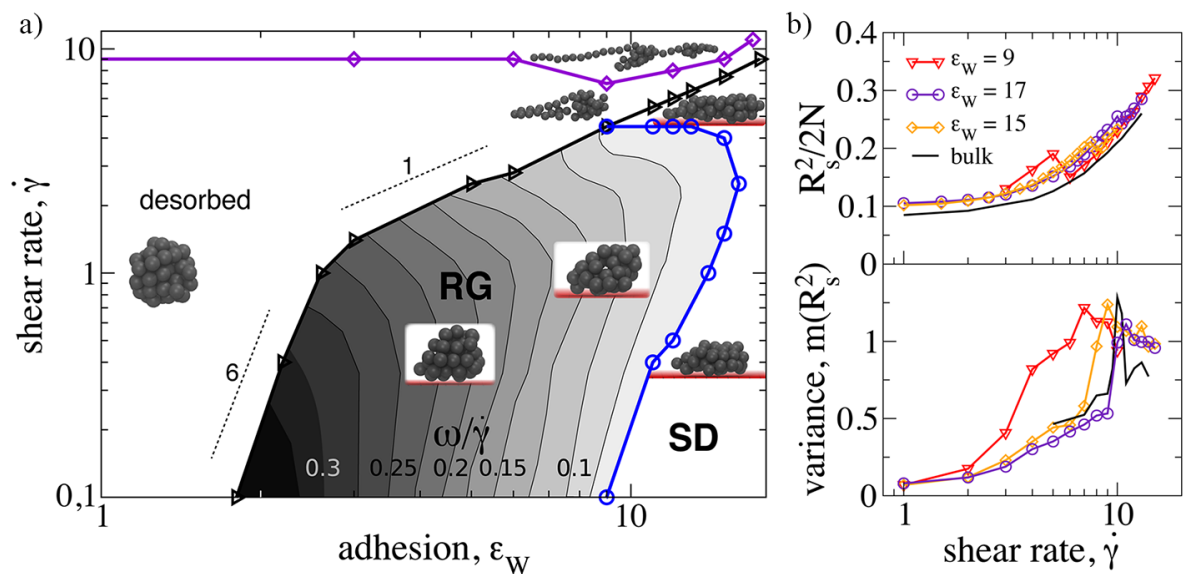

Fig. 4. a) Dynamic state diagram for a globule with fixed cohesive strength $\varepsilon=2$ at a homogeneous surface for varying shear rate $\dot{\gamma}$ and adhesive strength $\varepsilon_{W}$. The black line marks the desorption-adsorption transition. Slopes are indicated with dashed lines. The purple line denotes the stretch-transition at which the variance of the squared average extension $m\left(R_{s}^{2}\right)$ in (b) exhibits a peak. The transition from the rolling globule state (RG) to the slipping disk state (SD) is denoted by the blue line and defined by a value of the angular velocity of $\omega / \dot{\gamma}=0.05$. Snapshots show representative polymer configurations.

defined by the peak of the variance $m\left(R_{s}^{2}\right)$, denoted by the purple line in fig. $4 \mathrm{a}$, occurs for low adhesive strength at $\dot{\gamma}=9$, in agreement with previous studies in bulk shear flow [30].

\section{Results for homogeneous and hydrodynamically stagnant surface}

Local bonds between polymer monomers and discrete surface binding sites induce strong friction effects that considerably slow down lateral motion close to the surface, as we will discuss in detail in sect. 5. In this section, we elucidate the effects of high surface friction in a coarse grained model by imposing a reduced monomer mobility close to the laterally homogeneous surface, without actually resolving the discrete surface bindings sites. In the model we use in this section, the monomer self-mobility is reduced from a value of unity far away from the surface to a value of $\mu_{S}<1$ within a finite distance range from the surface, as described by eq. (7), equivalent to a hydrodynamically stagnant surface layer. Alternatively, one can think of the reduced self-mobility as being due to a layer of grafted polymers.

In the following we concentrate on the effect of this reduced surface mobility on the globule adsorption transition for small shear rates. In the main part of fig. 5 we compare the adsorption transition for fixed cohesive strength $\varepsilon=2$ for the case of homogeneous mobility $\mu_{s}=1$ (solid lines) with the case of stagnant surface mobility $\mu_{s}=0.01$ (dashed lines). The latter parameter value means that the monomer mobility is reduced by a factor of 100 close to the surface. For each surface mobility scenario we show results for free-draining (FD, gray lines) simulations and for simulations including hydrodynamic interactions (HI, black lines). The HI result for $\mu_{s}=1$ thus agrees with the adsorption transition already shown in fig. 4a. Note that

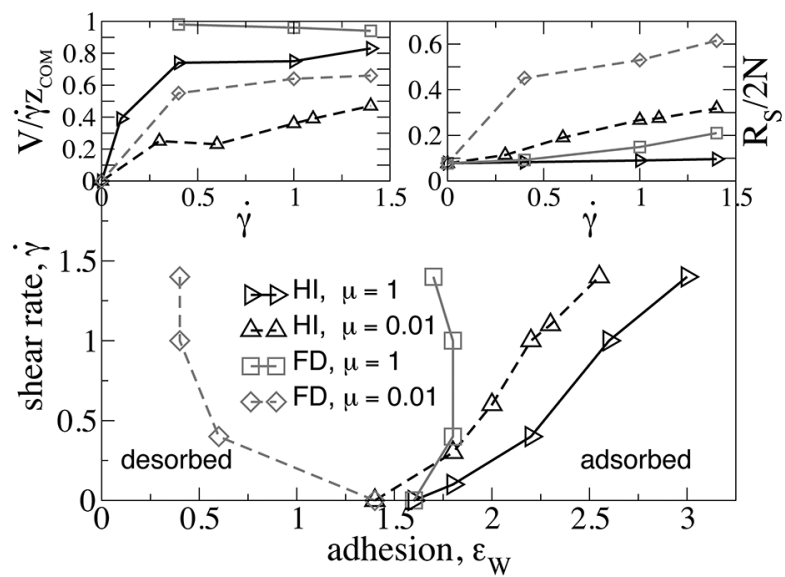

Fig. 5. Results for a homogeneous surface with stagnant boundary layer. The dynamic state diagram shows the adsorption transition for a globule with fixed cohesive strength $\varepsilon=2$ on a surface with potential range $\sigma_{W}=1.5$. Black symbols include hydrodynamic interactions (HI); gray symbols are results from free-draining (FD) simulations. For solid lines the monomers mobility is homogeneous $\left(\mu_{s}=1\right)$, dashed lines show results for a stagnant surface layer within which the monomer mobility is reduced to one percent of the bulk value $\left(\mu_{s}=0.01\right)$. The insets show the rescaled velocity $V / \dot{\gamma} z_{\mathrm{COM}}$ and the extension $R_{S} / 2 N$ in flow direction as a function of the shear rate for all four scenarios, measured along the respective adsorption transitions displayed in the main figure.

under equilibrium condition, i.e. in the limit $\dot{\gamma}=0$, all four different models coincide within the numerical uncertainty.

Upon increasing the shear rate, we observe pronounced differences. In the presence of hydrodynamic interactions (black lines), an increase in shear rate induces desorption, which can be rationalized by the presence of hydrodynamic lift forces $[15,16]$. Going from a homogeneous mo- 
bility, $\mu_{s}=1$, to a stagnant mobility, $\mu_{s}=0.01$, only mildly changes the critical shear rate. We conclude that a surface friction that is increased by a factor of 100 enhances the adsorption tendency only very weakly.

In the free-draining case (FD) and for $\mu_{S}=1$ (gray solid line), the adhesive strength needed for adsorption does not vary much with the shear rate, the adsorption transition is almost vertical. This reflects the absence of lift forces that would counteract adhesive forces. For the FD case with a reduced surface mobility $\mu_{s}=0.01$ (gray broken line), we observe pronounced shear-induced adsorption of the globule; i.e., the adsorption transition shifts towards lower $\varepsilon_{W}$. In the insets we show results for the average extension in flow direction $R_{S} / 2 N$ and for the chain velocity rescaled by the unperturbed fluid velocity at the center-of-mass position $V / \dot{\gamma} z_{\mathrm{COM}}$, measured along the adsorption transition line. One sees that the rescaled velocity is unity only in the free-draining case (FD) and for $\mu_{S}=1$ (gray solid line), both surface friction and hydrodynamic interactions reduce the chain velocity. The velocity reduction leads to a pronounced chain elongation, which promotes adsorption due to the reduced chain fluctuations [34]. In the FD case this gives rise to shear-induced adsorption, in the HI case this effect is overwhelmed by hydrodynamic lift forces and therefore shearinduced adsorption is not observed.

\section{Results for inhomogeneous surface with discrete binding sites}

\subsection{Fixed shear rate, variable corrugation}

Next we investigate how a more realistic surface binding model changes the adsorption behavior. We consider an inhomogeneous surface consisting of discrete binding sites, represented by Lennard-Jones centers, arranged on a square lattice with lattice constant $b=\tilde{b} / a=2$ and located on a plane at position $z_{W}=2$. In order to change the degree of corrugation, we vary the interaction range $\sigma_{W}<b$ defined in eq. (4) while keeping the density of binding sites constant. For lower surface binding site density, the critical adhesive strength for adsorption increases but otherwise the character of the adsorption transition does not change (data not shown). The important parameter in this section is the ratio of the interaction range and the lattice constant, $\sigma_{W} / b$, which controls the effective mobility of a monomer on the surface.

In fig. 6 we show the boundary between the adsorbed and the desorbed state for a globule with fixed cohesive strength of $\varepsilon=2$ and for a fixed shear rate $\dot{\gamma}=1$ as a function of the surface site interaction range $\sigma_{W}$ and the adhesive strength $\varepsilon_{W}$. We compare free-draining simulations (FD, gray solid line) with hydrodynamic simulations (HI, black solid line) and also show equilibrium results in the absence of shear $(\dot{\gamma}=0$, gray broken line). All three curves show that the critical adhesive strength increases in order to compensate for a shorter interaction range. When we compare the two FD curves, we see that a finite

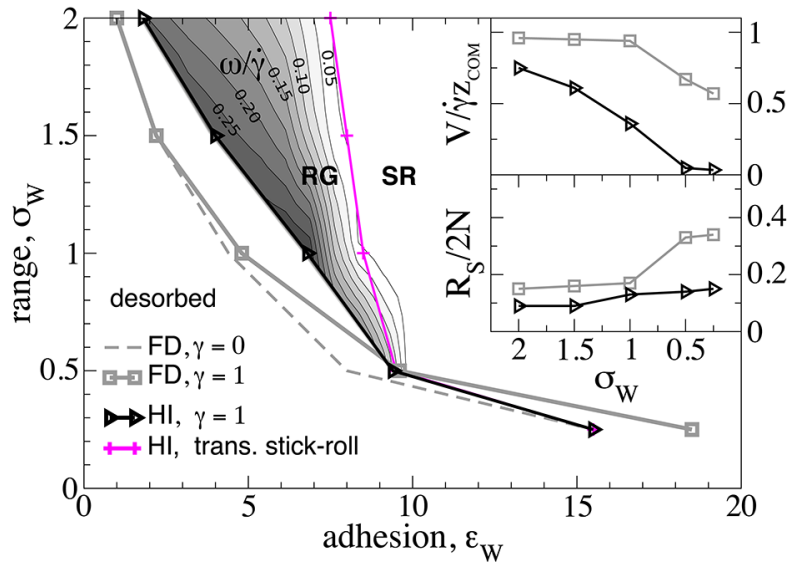

Fig. 6. Dynamic state diagram for a globule with fixed cohesive strength $\varepsilon=2$ at an inhomogeneous surface as a function of the adhesive strength $\varepsilon_{W}$ and the interaction range $\sigma_{W}$ of the binding sites. The solid black and gray lines denote the adsorption transition at fixed shear rate $\dot{\gamma}=1$ in the presence (black) and absence (gray) of hydrodynamic interactions. For comparison, the dashed gray line denotes the equilibrium adsorption transition without shear, $\dot{\gamma}=0$. The transition from the rolling globule state (RG) to the stick-roll state (SR) is denoted by the magenta line and defined by a maximum in the variance of the squared translational velocity $m\left(V^{2}\right)$, see fig. $7 \mathrm{~b}$. The underlying contour plot shows the angular velocity $\omega / \dot{\gamma}$. The transition to the stick-roll state approximately coincides with the previous criterion for the roll-slip transition $\omega / \dot{\gamma}=0.05$. The insets show the rescaled velocity $V / \dot{\gamma} z_{\mathrm{COM}}$ and the extension $R_{S} / 2 N$ as a function of $\sigma_{W}$ measured along the respective adsorption transition lines.

shear flow ( $\dot{\gamma}=1$, solid gray line) slightly weakens the adsorption and shifts the transition towards higher values of $\varepsilon_{W}$. As can be seen in the insets of fig. 6 , the rescaled velocity $V / \dot{\gamma} z_{\mathrm{COM}}$, measured along the adsorption transition line, decreases with decreasing interaction range $\sigma_{W}$ as a result of enhanced surface friction, while the extension in flow direction $R_{S} / 2 N$, also measured along the transition line, increases strongly due to internal shear effects in the globule. This means that the inhomogeneous surface enhances unfolding of the FD polymer in shear flow but suppresses adsorption; in other words, the stretching of the chain, which by itself weakens entropic repulsion from the surface and therefore promotes adsorption [34], is overwhelmed by the weakened energetic attraction to the surface since the monomers are constantly pulled away from the surface binding sites $[17,14]$.

In the presence of hydrodynamic interactions (solid black line in fig. 6) hydrodynamic lift forces additionally weaken the adsorption propensity. Due to the decreased monomer self-mobility at the surface, the globule velocity $V / \dot{\gamma} z_{\mathrm{COM}}$ (black line in inset fig. 6) is further reduced when compared with the FD case (gray line). Hydrodynamic screening effects that are connected to the flow stagnation inside the globule reduce the chain elongation $R_{S} / 2 N$ when compared with FD simulations (black and gray lines in lower inset fig. 6). The contour plot of the 


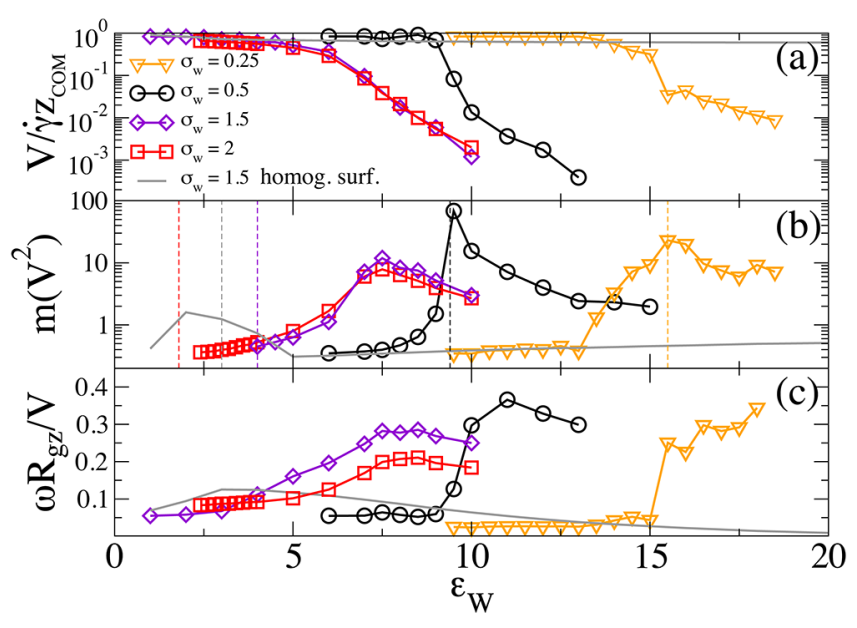

Fig. 7. Results for the inhomogeneous surface for a few different values of the binding site interaction range $\sigma_{W}$ for fixed $\epsilon=2$ and $\dot{\gamma}=1$. For comparison, we include results for the homogeneous surface (gray lines). a) The rescaled translational velocity $V / \dot{\gamma} z_{\mathrm{COM}}$ decreases over several orders of magnitude with increasing $\epsilon_{W}$. b) The variance of the squared average velocity $m\left(V^{2}\right)$ shows a peak which defines the transition to the stick-roll state. Dashed vertical lines mark the globule adsorption transition. c) Although both angular and translational velocities are very small in the stick-roll state, the ratio $\omega R_{g z} / V$ is of the order of unity and thus indicative of intermittent rolling motion.

rescaled angular velocity $\omega / \dot{\gamma}$ in fig. 6 illustrates that the globule rolling motion is essentially arrested for short interaction ranges $\sigma_{W} \leq 0.5$. As a result, short ranges lead to an adsorption transition at an adhesive strength comparable to the equilibrium case. As opposed to the case of a homogeneous surface, where we observe a transition to a slipping state, for the inhomogeneous surface we observe a transition to stick-roll motion, indicated by the magenta line in fig. 6 , which is defined by a peak in the variance of the squared globule velocity $m\left(V^{2}\right)$ in fig. $7 \mathrm{~b}$. Note that this criterion almost exactly coincides with our previous definition of the transition from rolling to slipping, $\omega / \dot{\gamma}=0.05$.

\subsection{The stick-roll state}

In fig. 7 we plot the average rescaled velocity $V / \dot{\gamma} z_{\mathrm{COM}}$, the variance $m\left(V^{2}\right)$, defined similarly to eq. (8), as well as the ratio of the rescaled angular and translational velocities $\omega R_{g z} / V$ for a few fixed values of the interaction range $\sigma_{W}$ as a function of the surface adhesion strength $\varepsilon_{W}$. While $V / \dot{\gamma} z_{\mathrm{COM}}$ in fig. 7a decreases over several orders of magnitude upon increasing $\varepsilon_{W}$, the variance $m\left(V^{2}\right)$ in fig. 7b shows a well-defined peak which defines the transition to the stick-roll state (SR). For short interaction ranges $\sigma_{W} \leq 0.5$ this transition coincides with the adsorption transition, denoted in fig. $7 \mathrm{~b}$ by dashed vertical lines, which means that for a highly corrugated surface potential an adsorbed globule is always in the stick-roll state. In fig. 7 we also include results for the homogeneous surface for $\sigma_{W}=1.5$ (gray line). For this case the average velocity in fig. 7a approaches a constant value of about $V / \dot{\gamma} z_{\mathrm{COM}}=0.6$ and the variance $m\left(V^{2}\right)$ in fig. $7 \mathrm{~b}$ is rather small, only displaying a weak peak at the adsorption transition. For the inhomogeneous surface the ratio $\omega R_{g z} / V$ in fig. 7c displays a maximum for large adhesion $\varepsilon_{W}$ meaning that angular and translational velocities are synchronized. For the homogeneous surface, on the other hand, we observe slipping at large $\varepsilon_{W}$ meaning that the angular velocity is much lower than the translational velocity.

The transition from rolling to stick-roll motion is further illustrated by time series in fig. 8a for fixed $\sigma_{W}=1.5$ and for three different values of the adhesive strength $\varepsilon_{W}$ that all correspond to adsorbed globule states. Only weak friction effects are observed for $\varepsilon_{W}=5$, where the globule continuously moves over the surface with modest relative fluctuations of the velocity $V$ (black line) around its mean and the displacement $x_{\mathrm{COM}}$ (red line) continuously increases with time, similar to the homogeneous surface. Deformations of the globule are weak; the extension $R_{S} / 2 N$ (gray line) is small and almost constant. For $\varepsilon_{W}=8$ the maximal variance $m\left(V^{2}\right)$ is reached (compare fig. 7b). Accordingly, the velocity $\mathrm{V}$ in fig. 8a shows pronounced peaks and the globule is temporarily stuck on the surface, indicated by plateaus in $x_{\mathrm{COM}}(\mathrm{red})$. The close correlations between peaks in the velocity and the angular velocity $\omega / \dot{\gamma}$ lead to the conclusion that the polymer moves by instantaneous rolling rather than slipping events, hence the name stick-roll state. The plateaus in the extension $R_{S} / 2 N$ (gray line) suggest that the rolling events are accompanied by unwinding and refolding of the polymer. Increasing the adhesion even further to $\varepsilon_{W}=10$ extends the periods over which the globule is stuck on the surface.

In fig. $8 \mathrm{~b}$ we show auto-correlation functions of the translational velocity $C_{V V}(t)$, defined by eq. (10). For a globule in the rolling globule (RG) state for $\varepsilon_{W}=5$, the velocity auto-correlation decays quickly. For a polymer in the stick-roll (SR) state for $\varepsilon_{W}=10, C_{V V}(t)$ decays even faster and exhibits an anti-correlated regime, indicative of alternating switching between periods of motion and periods of arrest on the surface. The longest correlation time is observed for intermediate adhesion $\varepsilon_{W}=8$, reflecting that this is the location of a transition from rolling to stick-roll behavior.

\subsection{Variable shear rate, fixed corrugation}

We next study the adsorption behavior for fixed range of the surface binding sites $\sigma_{W}=0.5$ and variable shear rate $\dot{\gamma}$; as before we fix the cohesive strength at $\varepsilon=2$. In fig. 9a we show the state diagram including the adsorption transition (black line). The diagram is similar as for the homogeneous surface in fig. 4, the higher the shear rate, the stronger the critical adhesive strength; i.e., shear promotes desorption. Included are typical globule snapshots at parameters denoted by the adjacent labels. The globule, 
a)

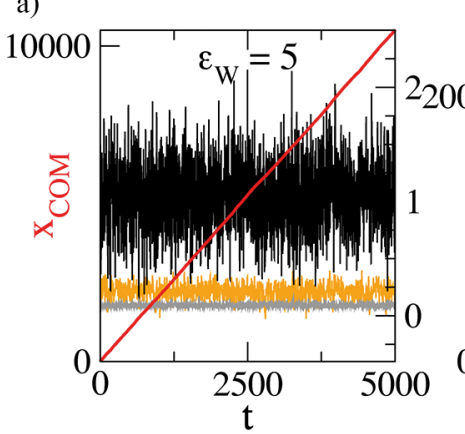

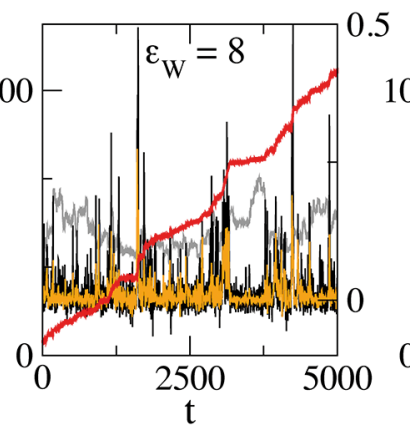

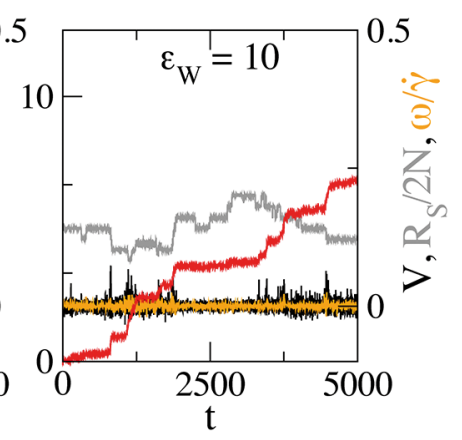

b)

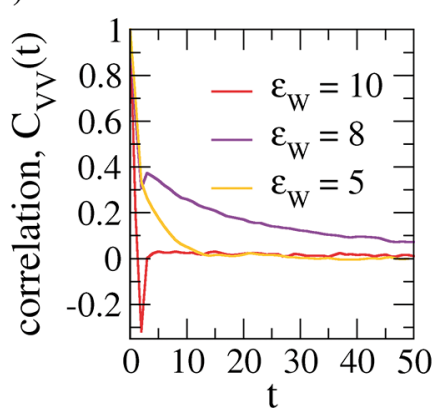

Fig. 8. a) Time series at an inhomogeneous surface with binding site potential range $\sigma_{W}=1.5$ for fixed $\epsilon=2$ and $\dot{\gamma}=1$. For the different values $\varepsilon_{W}=5,8,10$ we show the chain displacement in flow direction $x_{\mathrm{COM}}$ (red), the translational velocity $V$ (black), the angular velocity $\omega / \dot{\gamma}$ (orange) and the chain extension $R_{S} / 2 N$ (gray). While for $\varepsilon_{W}=5$ the globule rolls continuously like on a homogeneous surface, for $\varepsilon_{W}=8$ one sees pronounced steps in the displacement and intermittent peaks in the velocity. For $\varepsilon_{W}=10$, the polymer is arrested over extended periods of time. b) Velocity-velocity auto-correlation functions.
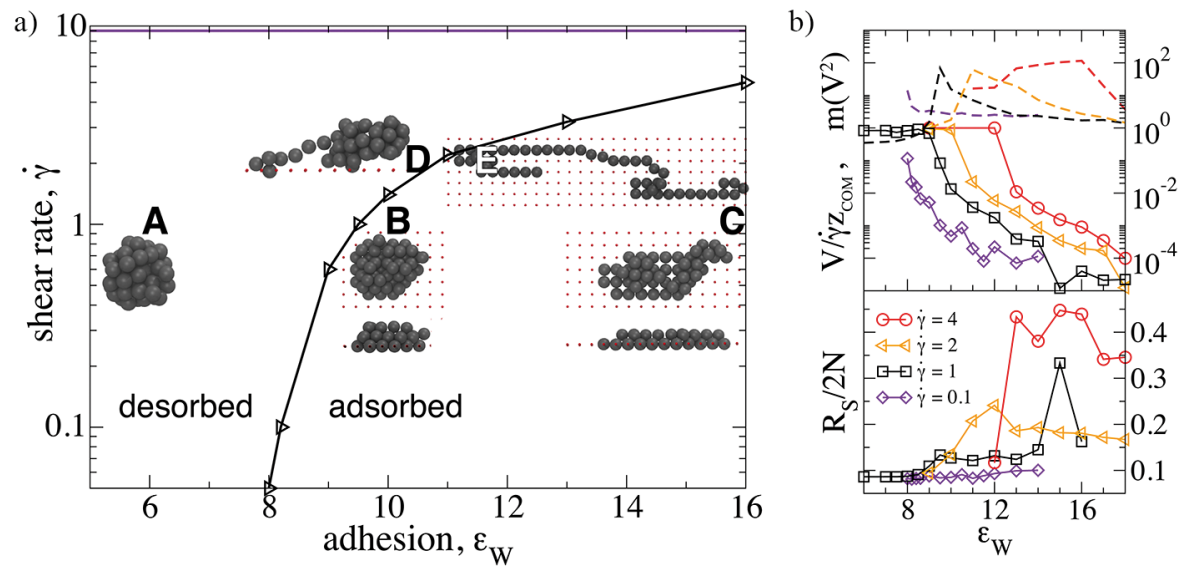

Fig. 9. a) Dynamic state diagram for a globule with fixed cohesive strength $\varepsilon=2$ at an inhomogeneous surface with fixed binding site potential range $\sigma_{W}=0.5$ as a function of shear rate $\dot{\gamma}$ and adhesive strength $\varepsilon_{W}$. The black line marks the desorptionadsorption transition. The stretch transition occurs in the desorbed state and is denoted by the purple line. Snapshots (top and side views) show representative polymer configurations for $\dot{\gamma}=1$ (A-C) and for $\dot{\gamma}=2$ (D,E), where red dots represent surface binding sites. b) The drastic decrease of the rescaled translational velocity $V / \dot{\gamma} z_{\mathrm{COM}}$ (solid lines) in the adsorbed state and the large variance $m\left(V^{2}\right)$ (dashed lines) indicate stick-roll motion. Due to deformation and unfolding at high shear rates, the average extension $R_{S}$ increases.

which exhibits a spherical shape in the desorbed state, see snapshot (A), adsorbs for $\dot{\gamma}=1$ at an adhesive strength $\varepsilon_{W}=9.5$ and exhibits a disk-like conformation, see (B). The peak of $m\left(V^{2}\right)$ for $\dot{\gamma}=1$ in fig. 9b occurs roughly at $\varepsilon_{W}=9.5$ and shows that the adsorbed state corresponds to the stick-roll state. The average velocity $V / \dot{\gamma} z_{\mathrm{COM}}$ in the adsorbed state is reduced by orders of magnitude compared to the homogeneous surface. The average extension $R_{S} / 2 N$ for $\dot{\gamma}=1$ only slightly increases with increasing adhesive strength $\varepsilon_{W}$, as seen in the snapshot $(\mathrm{C})$ and also in fig. 9 b. At higher shear rate $\dot{\gamma}=2$ stronger deformations are obtained, see snapshot (E). The snapshot (D) shows a stretched state where only few beads are in contact with the surface; in fact, snapshot (D) is taken just before the polymer desorbs.

\section{Summary and conclusion}

In the first part we investigate the adsorption behavior of a globule at a homogeneous surface for varying cohesive and adhesive strengths and shear rates. The dynamic state diagram features different rolling and slipping states: the rolling globule state, the rolling prolate state, and the rolling folding-unfolding state. Slipping globules are observed for large adhesion and slipping coil conformation are found for low cohesion. Shear does not promote or enhance adsorption. For non-zero shear, we identify two cyclic processes in terms of the chain elongation and the separation from the surface.

In the second part surface friction effects are accounted for by a hydrodynamically stagnant surface layer, within which the mobility is reduced. This results in a local veloc- 
ity reduction of the chain and thus to increased elongation in flow direction. While this leads to a shear-induced adsorption in the freely draining situation, in the presence of hydrodynamic interactions the stagnant surface layer does not change the adsorption behavior drastically. We next consider an inhomogeneous surface consisting of discrete binding sites having a variable interaction range. The resulting surface friction leads to a drastic velocity reduction when including full hydrodynamic interactions. Although this reduces the hydrodynamic lift force, the effective surface adhesion strength is reduced so that adsorption is disfavored when compared to the limit of no shear. This is similar to the analysis of a simple model of a monomer dragged along a corrugated potential [17].

One result of our work is that for a simple interaction model based on a static Hamiltonian consisting of pair potentials between monomers and the surface, the globule adsorption is not enhanced by the presence of shear, leading to the tentative conclusion that in order to observe such a behavior, a more complex binding mechanism including saturating or catch-bonds [3] is needed, in line with previous results [19]. This in turn suggests to construct more complex potential-based binding models for the interaction of collapsed proteins with surfaces or with colloidal particles in shear, in order to model biological important situations such as the adsorption of the von Willebrand factor on vessel walls in shear [5] and the flowdriven polymer-colloid composite formation [35]. Hence, in future work the von Willebrand domain structure could be resolved by a more detailed coarse grained heteropolymer model. Another interesting question is how catch-bond behavior, which in previous simulation work has been implemented in Langevin simulations by a heuristic two-state reaction bond model [19], can be modeled in a purely potential-based Langevin simulation.

The authors thank Alfredo Alexander-Katz, Frauke Gräter, Joachim Rädler, and Matthias Schneider for stimulating discussions and acknowledge financial support from the DFG Research Unit FOR 1543.

Open Access This is an open access article distributed under the terms of the Creative Commons Attribution License (http://creativecommons.org/licenses/by/4.0), which permits unrestricted use, distribution, and reproduction in any medium, provided the original work is properly cited.

\section{References}

1. R.R. Netz, D. Andelman, Phys. Rep. 380, 1 (2003).

2. C.B. Korn, U.S. Schwarz, Phys. Rev. E 77, 041904 (2008).

3. W.E. Thomas, V. Vogel, E. Sokurenko, Annu. Rev. Biophys. 37, 399 (2008).
4. R.P. McEver, C. Zhu, Annu. Rev. Cell Dev. Biol. 26, 363 (2010).

5. S.W. Schneider, S. Nuschele, A. Wixforth, C. Gorzelanny, A. Alexander-Katz, R.R. Netz, M.F. Schneider, Proc. Natl. Acad. Sci. U.S.A. 104, 7899 (2007).

6. T.A. Springer, J. Thromb. Haemost. 9, 130 (2011).

7. Z.M. Ruggeri, J. Thromb. Haemost. 1, 1335 (2003).

8. E. Eisenriegler, K. Kremer, K. Binder, J. Chem. Phys. 77, 6296 (1982).

9. A. Milchev, K. Binder, Macromolecules 29, 343 (1996).

10. R. Descas, J.-U. Sommer, A. Blumen, J. Chem. Phys. 120, 8831 (2004)

11. M. Möddel, W. Janke, M. Bachmann, Macromolecules 44, 9013 (2011).

12. G.L. He, R. Messina, H. Löwen, J. Chem. Phys. 132, 124903 (2010).

13. N. Hoda, S. Kumar, J. Chem. Phys. 127, 234902 (2007).

14. N. Hoda, S. Kumar, J. Chem. Phys. 128, 164907 (2008).

15. C. Sendner, R.R. Netz, EPL 79, 58004 (2007).

16. C. Sendner, R.R. Netz, EPL 81, 54006 (2008).

17. A. Serr, C. Sendner, F. Müller, T.R. Einert, R.R. Netz, EPL 92, 38002 (2010).

18. C.E. Sing, A. Alexander-Katz, EPL 95, 48001 (2011).

19. C.E. Sing, J.G. Selvidge, A. Alexander-Katz, Biophys. J. 105, 1475 (2013).

20. D.E. Smith, H.P. Babcock, S. Chu, Science 283, 1724 (1999).

21. J.R. Blake, Proc. Camb. Phil. Soc. 70, 303 (1971).

22. Y. von Hansen, M. Hinczewski, R.R. Netz, J. Chem. Phys. 134, 235102 (2011).

23. B. Dünweg, A.J.C. Ladd, in Advanced Computer Simulation Approaches for Soft Matter Sciences III, Vol. 221 of Adv. Polym. Sci. (Springer Berlin Heidelberg, 2009) pp. $89-166$.

24. G. Gompper, T. Ihle, D.M. Kroll, R.G. Winkler, in $A d-$ vanced Computer Simulation Approaches for Soft Matter Sciences III, Vol. 221 of Adv. Polym. Sci. (Springer Berlin Heidelberg, 2009) pp. 1-87.

25. C.E. Sing, A. Alexander-Katz, Biophys. J. 98, L35 (2010).

26. C.E. Sing, A. Alexander-Katz, J. Chem. Phys. 135, 014902 (2011).

27. M. Doi, S.F. Edwards, The theory of polymer dynamics, Vol. 73 (Oxford University Press, 1988).

28. A.J. Goldman, R.G. Cox, H. Brenner, Chem. Eng. Sci. 22, 653 (1967).

29. M. Kraus, W. Wintz, U. Seifert, R. Lipowsky, Phys. Rev. Lett. 77, 3685 (1996).

30. A. Alexander-Katz, M.F. Schneider, S. Schneider, A. Wixforth, R.R. Netz, Phys. Rev. Lett. 97, 138101 (2006).

31. A. Alexander-Katz, R.R. Netz, Macromolecules 41, 3363 (2008).

32. A. Alexander-Katz, R.R. Netz, EPL 80, 18001 (2007).

33. C. Sendner, R.R. Netz, Eur. Phys. J. E 30, 75 (2009).

34. A. Serr, R.R. Netz, EPL 78, 68006 (2007).

35. H. Chen, M.A. Fallah, V. Huck, J.I. Angerer, A.J. Reininger, S.W. Schneider, M.F. Schneider, A. AlexanderKatz, Nat. Commun. 4, 1333 (2013). 